\title{
Is there a place for Ramucirumab after Sorafenib in patients with advanced HCC?
}

\author{
Yeo Jeong So ${ }^{1}$, Sophia Frentzas ${ }^{1,2}$, Eva Segelov ${ }^{1,2}$ \\ ${ }^{1}$ Medical Oncology, Monash Health, Melbourne, Australia; ${ }^{2}$ Translational Oncology Research Laboratory, Monash University, Melbourne, Victoria, Australia \\ Correspondence to: Professor Eva Segelov. Monash Health and Monash University, Melbourne, Victoria, Australia. Email: eva.segelov@monash.edu. \\ Comment on: Zhu AX, Kang YK, Finn RS, et al. Ramucirumab after sorafenib in patients with advanced hepatocellular carcinoma and increased \\ $\alpha$-fetoprotein concentration (REACH-2): a randomized, double blind, placebo-controlled, phase-3 trial. Lancet Oncol 2019;20:282-96.
}

Submitted Apr 10, 2019. Accepted for publication Apr 19, 2019.

doi: $10.21037 /$ hbsn.2019.04.14

View this article at: http://dx.doi.org/10.21037/hbsn.2019.04.14

Hepatocellular carcinoma (HCC) is the sixth most common incident and the fourth most common cause of cancer death worldwide. It is strongly associated with chronic Hepatitis $\mathrm{B}$ and $\mathrm{C}$ infection and excess alcohol intake (1), making treatment of patients with HCC complicated, with the almost uniform presence of concurrent cirrhosis and varying degrees of liver failure, which often fluctuates over time.

HCC has a complex molecular pathogenesis, with angiogenesis identified as a critical hallmark. As such, this gave rise to the development of the anti-cancer class of therapeutics targeting the vascular endothelial growth factor receptor (VEGF-R). Sorafenib, the oral small molecule multikinase inhibitor against VEGFR isoforms $1-3$ and platelet derived growth factor receptor $\beta$, was the sole FDA approved, first line therapy for advanced disease for the past 10 years $(2,3)$, until lenvatinib was approved in 2018 on the basis of non-inferiority (4) (Table 1).

In the second line setting, four agents are now FDA approved, all trialed in patients who had progressed on, or were intolerant of, sorafenib. The oral multikinase inhibitors regorafenib and cabozantinib each showed improved survival over placebo $(5,6)$ (Table 1), whereas the PD-1 inhibitors, nivolumab and pembrolizumab, were approved based on efficacy in open labeled Phase II trials (7). Both are currently undergoing evaluation in Phase III studies against best supportive care.

Ramucirumab, a recombinant IgG1monoclonal antibody targeting VEGFR-2, has shown efficacy in various tumours, although this appears to be dependent on line of therapy, at least in some cancers such as gastric. Its use as second line therapy in HCC was investigated in the Phase III randomized controlled REACH trial of 565 sorafenib pretreated patients. Although there were modest improvements over placebo in the secondary endpoints of progression-free survival (PFS), time to tumour progression and objective response, the primary endpoint of overall survival (OS) was not met in the intention-to-treat population [hazard ratio (HR) 0.71, $\mathrm{P}=0.14$ ] (8).

However, there was an interesting observation in the pre-specified subgroup of patients with alpha-fetoprotein $(\mathrm{AFP})>400 \mathrm{ng} / \mathrm{mL}$, present in approximately $50 \%$ of participants. Elevated AFP is a recognized poor prognostic factor and has been associated with increased angiogenesis and VEGFR expression (9). In this subgroup, ramucirumab was associated with a significant OS improvement of 3.5 months (HR 0.67, $\mathrm{P}=0.006$ ). This hypothesis-generating finding was definitively tested in the REACH-2 trial, in which 292 patients from 20 countries, with Barcelona Clinic Liver Cancer (BCLC) stage B or C advanced HCC, ECOG performance status 0 or 1, Child-Pugh class A liver disease and AFP >400 ng/mL, were randomized in a 2:1 design to ramucirumab $8 \mathrm{mg} / \mathrm{kg}$ intravenously second weekly or placebo.

Zhu et al. published results after a median follow-up for OS (the primary endpoint) of 7.6 months, at which point 206 patients $(70 \%)$ had progressed and 221 patients (76\%) had died. Median OS was significantly improved for ramucirumab versus placebo [8.5 months $(95 \% \mathrm{CI}$, 7.0-10.6) vs. 7.3 months (5.4-9.1); HR 0.71 (95\% CI, 0.531-0.949); $\mathrm{P}=0.02]$. The benefit appeared to commence after approximately three months of treatment and increased over time, with all subgroups appearing to 
Table 1 Pivotal trials in advanced hepatocellular carcinoma (2,4-6)

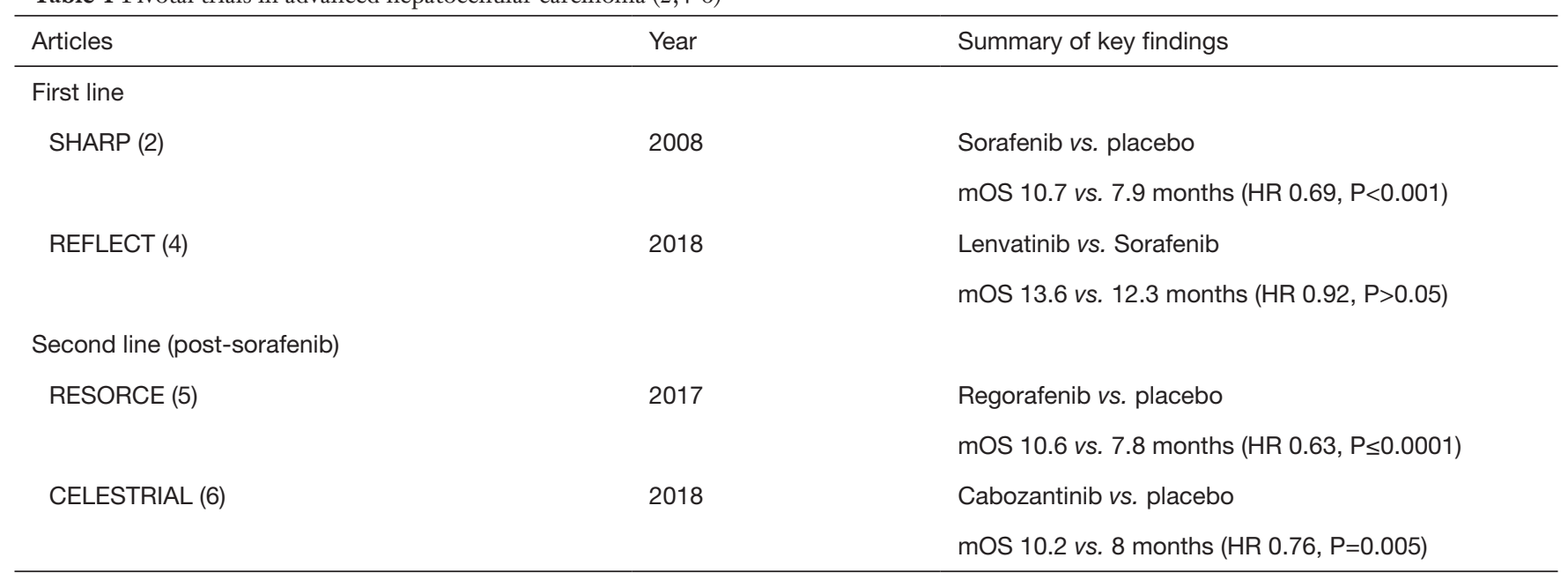

benefit (apart from the very low number of females in the placebo group). Median PFS was also significantly longer at 2.8 months (95\% CI, 2.8-4.1) vs. 1.6 months (1.5-2.7), HR 0.45 (95\% CI, 0.34-0.60); $\mathrm{P}<0.0001$. Toxicity was similar to that in REACH and was deemed acceptable and patient reported outcomes were similar between groups. A preplanned pooled individual patient data analysis with the AFP >400 ng/mL subgroup from REACH showed a survival benefit of similar magnitude.

How should this data be utilized in the management of patients with refractory HCC? The findings raise the tantalizing hypothesis of a biologically different subgroup within HCC, with an easy-to-measure predictive biomarker. There are some cautions to note, however. AFP levels are a continuum, so that an outcome difference based on a single threshold is implausible, as is the implication that a threshold dictates different biology; if it is a surrogate marker then how exactly does AFP correlate with the distinct phenotype? The REACH-2 trial also had a longerthan-expected survival for the relatively small placebo group, possibly attributed to a chance imbalance in baseline AFP, although other unrecognized factors could have contributed. Finally, the criteria for trial entry were tight, including exclusion of patients with clinically significant ascites, limiting applicability to patients encountered in real world practice.

$\mathrm{Up}$ against now numerous other treatment options for patients with refractory HCC, none of which have been compared head to head, ramucirumab provides an extra choice for physicians and some patients, who can together consider preference based on mode of delivery, toxicity and cost. Yet various questions remain: can ramucirumab benefit, in the high AFP population after sorafenib, be extrapolated to treatment after lenvatinib? Do we need to repeat $2^{\text {nd }}$ line trials when $1^{\text {st }}$ line therapy changes, as it may well further do with trials of upfront checkpoint inhibitors underway? On the other end of the clinical journey, can we extrapolate similar benefit for ramucirumab in later than $2^{\text {nd }}$ line settings e.g., after immunotherapy? Or like the renal cell cancer paradigm, should we start combining immunotherapy and targeted agents and if so, how should we trade increased toxicity for potential increased benefits? What now is the appropriate place in the treatment sequence for palliative locoregional therapies?

As the landscape of advanced HCC changes rapidly from a time not too far distant of little active systemic therapy, it is beholden on us to undertake carefully thought-out trials with as much correlative biospecimen collection and analysis as possible. Only in this way will we be able to finally select the right treatment for the right patient at the right time.

\section{Acknowledgments}

None

\section{Footnote}

Conflicts of Interest: The authors have no conflicts of interest to declare. 


\section{References}

1. Global Burden of Disease Liver Cancer Collaboration, Akinyemiju T, Abera S, et al. The burden of primary liver cancer and underlying etiologies from 1990 to 2015 at the global, regional, and national level: Results from the Global Burden of Disease Study 2015. JAMA Oncol 2017;3:1683-91.

2. Llovet JM, Ricci S, Mazzaferro V, et al. Sorafenib in advanced hepatocellular carcinoma. $\mathrm{N}$ Engl J Med 2008;359:378-90.

3. Cheng AL, Kang YK, Chen Z, et al. Efficacy and safety of sorafenib in patients in the Asia-Pacific region with advanced hepatocellular carcinoma: a phase III randomized, double-blind, placebo-controlled trial. Lancet Oncol 2009;10:25-34.

4. Kudo M, Finn RS, Qin S, et al. Lenvatinib versus sorafenib in first-line treatment of patients with unresectable hepatocellular carcinoma: a randomized phase 3 noninferiority trial. Lancet 2018;391:1163-73.

Cite this article as: So YJ, Frentzas S, Segelov E. Is there a place for Ramucirumab after Sorafenib in patients with advanced HCC? HepatoBiliary Surg Nutr 2019;8(5):546-548. doi: 10.21037/hbsn.2019.04.14
5. Bruix J, Qin S, Merle P, et al. Regorafenib for patients with hepatocellular carcinoma who progressed on sorafenib treatment (RESORCE): a randomized, double blind, placebo-controlled, phase 3 trial. Lancet 2017;389:56-66.

6. Abou-Alfa GK, Meyer T, Cheng AL, et al. Cabozantinib in patients with advanced and progressing hepatocellular carcinoma. N Engl J Med 2018;379:54-63.

7. Zhu AX, Finn RS, Edeline J, et al. Pembrolizumab in patients with advanced hepatocellular carcinoma previously treated with sorafenib (Keynote-224): a non-randomized, open-label phase 2 trial. Lancet Oncol 2018;19:940-52.

8. Zhu AX, Park JO, Ryoo BY, et al. Ramucirumab versus placebo as second line treatment in patients with advanced hepatocellular carcinoma following first line therapy with sorafenib (REACH): a randomized, double-blind, multicentre, phase 3 trial. Lancet Oncol 2015;16:859-70.

9. Shan YF, Huang YL, Xie YK, et al. Angiogenesis and clinicopathologic characteristics in different hepatocellular carcinoma subtypes defined by EpCAM and $\alpha$-fetoprotein expression status. Med Oncol 2011;28:1012-6. 